\title{
Fracture Shaft of Femur in Children with Newly Designed Femoral Brace
}

\author{
Mahara D P, Lamichhane A, Acharya P, Shrestha G C \\ Department of Orthopaedics, Tribhuvan University Teaching Hospital, Kathmandu, Nepal, Orthotist, Orthopedica, \\ Lalitpur, Nepal.
}

Correspondence address: Dr Deepak Prakash Mahara, Department of Orthopaedics, Tribhuvan University Teaching Hospital, Maharajgunj,Kathmandu, Nepal

Email: maharadeepak@yahoo.com

\begin{abstract}
Introduction: With the initial traction and secondary hip spica conservative treatment of fracture shaft of femur in children is an established technique of management all over the world. Three-six weeks of traction followed by another 6-8weeks of immobilization in spica cast at home takes away precious school going time of the children and professional earning time of the parents in our part of the world. The situation compelled us to think and design an Orthosis that not only splints the fractured femur but also maintains the continuous traction and acceptable alignment right from the beginning and allows early mobilization thereby avoiding need of long hospital stay, immobilization on spica cast and abstinence from school.

Methods: This Orthosis has been used successfully in 9 children with fractured shaft of femur, age ranged from 3 to 9 years with average age of 5 years. Initially the patients were put on skin traction. In the meantime, measurement of body parts was taken by an orthotist for fabrication of the brace, which was made available on second week. In presence of the orthotist the brace was applied and its fitting and pressure points were properly evaluated. The patients were discharged the next day and asked to follow up at 2,4,6,9 and 12 weeks with a roentgenogram at every visit. Patients were encouraged to bear weight with the affected limb with the brace on after achieving clinical union. Brace was removed after achieving radiological union.

Results: One patient did not turn up for follow up after application of brace. So, out of the ten patients, nine were included in this study as they had follow up of at least 12 weeks. Seven cases were male and two female. The average age of the patients was 5 years (range 3 to 9 years). The follow up period ranged from 12 weeks to 3 years with average period of 9.3 months. Radiological union was seen to occur at 12th week in 7 patients and 9th week in 2 patients and the brace was discarded at that time. Fracture united in all patients without any obvious angular, rotational deformities and limb length discrepancy.

Conclusion: Preliminary findings of this study showed the use of this Orthosis in the treatment of fracture shaft of femur in children has proved to be as good as the traditional method with distinct advantages of treating at home and early mobilization with the brace on.
\end{abstract}

Keywords: Spica cast, HKAFO, Fracture femur, Femoral brace 


\section{Introduction}

Treatment options for paediatric femoral fractures are many. Existing methods include spica casting with or without traction, cast bracing, external fixation, flexible or rigid intramedullary nailing, and plate fixation. The surgeon taking care of injured children should be comfortable with several techniques. Optimal treatment selection requires consideration of fracture, patient, and family factors. Traditionally, femur fractures in children of all ages had been treated by an initial period of traction, often until the appearance of callus on the radiograph, followed by a spica cast until solid union is achieved. The major disadvantages of spica casting are the result of prolonged immobilization or inability to control reduction in certain situations. Presence of the cast restricts access to and evaluation of soft-tissue injuries and skin integrity as dipicted in figure 1 .

Also such a prolonged hospitalization and immobilization may have significant implications such as; increased cost of treatment, scarcity of bed to other patients, loss of precious school going time of the children and professional earning time of the parents, especially in a developing country like ours. In adolescents, psychologic implications are especially important. Prolonged hospitalization alters the adolescent's self-image and interrupts social and educational development ${ }^{1}$.

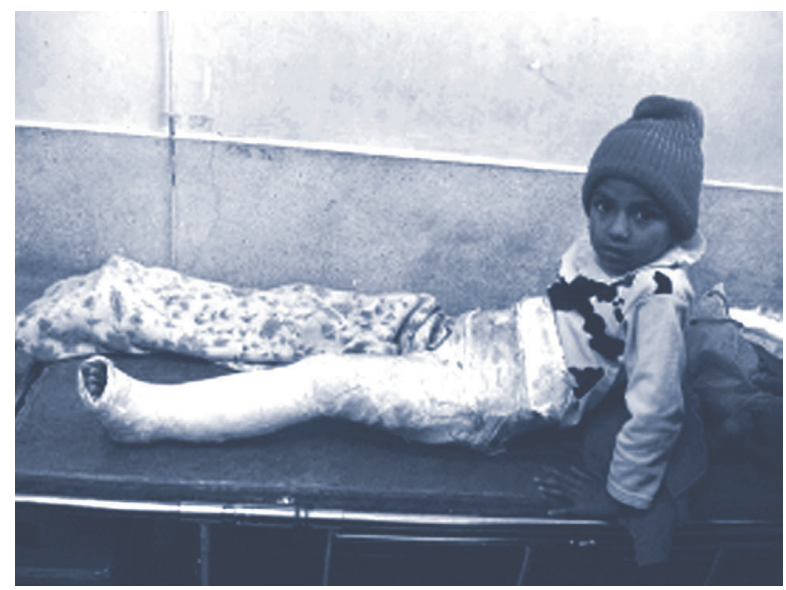

Figure 1 Boy with traditional hip spica having difficulty in finding a comfortable sitting position.

Taking advantage of the fact that fracture management in children is usually non-operative as they have high healing and remodeling potential (Rab GTet al. 2001) ${ }^{2}$, we chose to treat femoral fracture in children with a femoral brace rather than the traditional method ( traction followed by spica cast), thereby eliminating some of the demerits of spica cast. With this purpose we designed an Orthosis that not only splints the fractured femur but also maintains the continuous traction and acceptable alignment right from the beginning and allows early mobilization thereby avoiding need of long hospital stay, immobilization on spica cast and abstinence from school. The mainstay of this brace is the application of effective traction device in the form of telescoping femoral sections. This helps to maintain the femoral length, which is crucial for good functional outcome.

Our attempt is to see if we can achieve a similar if not a better result (in terms of fracture union) with less discomfort to children and their parents. The main purpose of this paper is to report the initial experience of the newly designed femoral brace treatment in our centre at Kathmandu, Nepal.

\section{Methods}

For the duration of 2 years ten patients agreed to be part of the study. Only nine patients had a minimum follow up period of 12 weeks. Children aged 2-12years with femoral diaphyseal fracture were included. Open and pathological fractures were excluded. Initially the patients were put on skin traction. In the meantime, measurement of body parts was taken by an orthotist for fabrication of the brace, which was made available on second week. In presence of the orthotist the brace was applied and its fitting and pressure points were properly evaluated. The neurovascular status was monitored and fracture alignment was assessed with a roentgenogram. Isometric exercise of thigh and leg muscles, and ambulation with crutches were taught to the older children. The patients were discharged the next day and asked to follow up at 2,4,6,9 and 12 weeks with a roentgenogram at every visit. Thereafter patients were called whenever necessary. At every visit clinicoradiological assessment was done to see the progress of union, angulation of fracture, shortening of the limb and other complications like pressure sores, breakage and loosening of orthosis. Patients were encouraged to bear weight with the affected limb with the brace on after achieving clinical union. Brace was removed after achieving radiological union.

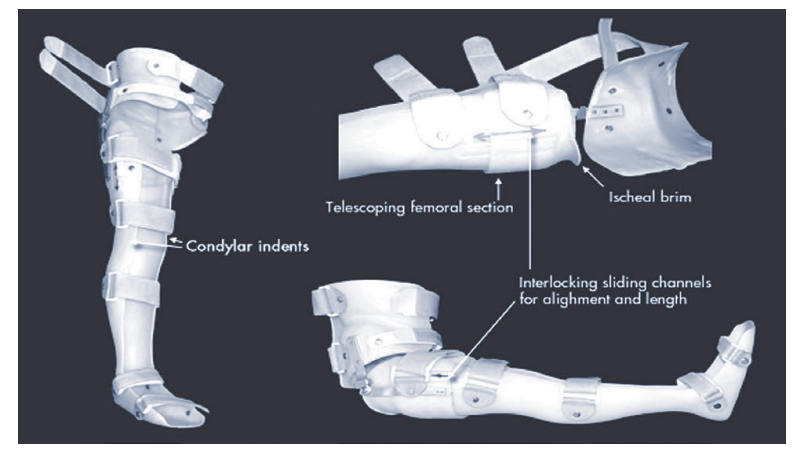

Figure 2 The Hip Knee Ankle Foot Orthosis (HKAFO) for the treatment of fracture shaft of femur in children, with its characteristic features. 
The Hip Knee Ankle Foot Orthosis (HKAFO) has a telescoping or adjustable femoral section (Figure 2). Longitudinal and rotational alignment of the femoral section is maintained by raised channels, which are vacuum formed in overlapping femoral section. Traction is maintained by the ischial brim (proximal) and supracondylar excavations which grips the medial and lateral condyles of the femur (distally). Rivets or screws in the femoral section maintain the length, which is determined by measuring the unaffected side. Overall rotational malalignment is eliminated by pelvic girdle, which is attached to the KAFO section by a hip joint with a drop lock. The knee is solid and the foot is incorporated to the brace for rotational alignment. Essentially this brace is a fixed traction device of femur which immobilizes the limb and prevents malrotation.

\section{Results}

One patient did not turn up for follow up after application of brace. So, out of the ten patients, nine were included in this study as they had follow up of at least 12 weeks as shown in table 1 .

Table 1: Characteristics of each patient with variables Seven cases were male and two female. early second week, however in two patients application of orthosis was delayed for 20 days due to fitting problem. All the patients were comfortable with the brace ( Figure $3 \mathrm{a}$ and $\mathrm{b}$ ). Patients and their parents were advised not to loosen any straps or try to remove the brace without supervision of orthotist or treating physician.

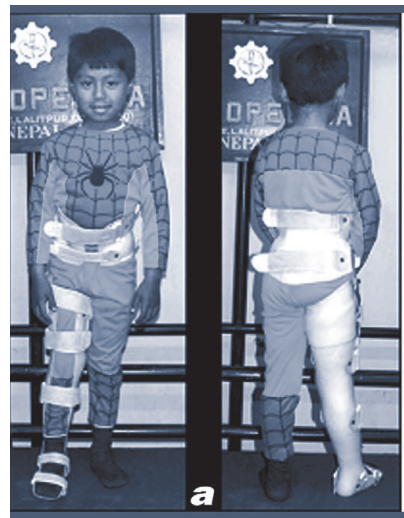

a.

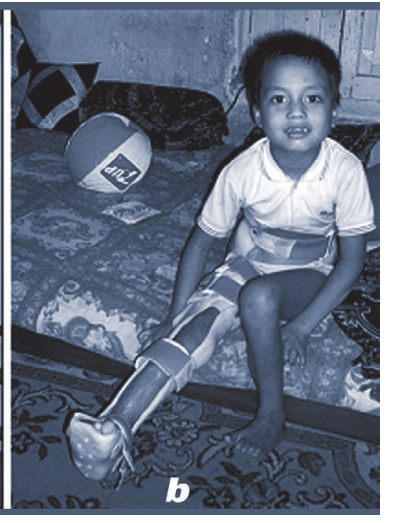

b.
Figure 3 (a). Five year old boy, (b) Four year old boy at his home.

\begin{tabular}{lllllllll} 
No. & Age(years) & Sex & Side & $\begin{array}{l}\text { Fracture } \\
\text { Pattern }\end{array}$ & $\begin{array}{l}\text { Traction } \\
\text { (days) }\end{array}$ & $\begin{array}{l}\text { Union } \\
\text { (weeks) }\end{array}$ & $\begin{array}{l}\text { Angulation } \\
\text { (degrees) }\end{array}$ & $\begin{array}{c}\text { Shortening } \\
\text { (cm) }\end{array}$ \\
\hline 1 & 4 & M & R & Oblique & 20 & 12 & None & None \\
\hline 2 & 4 & M & L & Oblique & 7 & 12 & None & None \\
\hline 3 & 5 & F & R & Spiral & 7 & 12 & None & None \\
\hline 4 & 9 & M & R & Oblique & 20 & 12 & $10^{\circ}$ varus & None \\
\hline 5 & 4 & M & L & Spiral & 7 & 12 & None & None \\
\hline 6 & 3 & F & R & Oblique & 7 & 9 & None & None \\
\hline 7 & 5 & M & R & Oblique & 10 & 12 & $10^{\circ}$ varus & None \\
\hline 8 & 8 & M & R & Transverse & 12 & 12 & None & 1 \\
\hline 9 & 3 & M & R & Spiral & 9 & 9 & None & None \\
\hline
\end{tabular}

The average age of the patients was 5 years (range 3 to 9 years). The follow up period ranged from 12 weeks to 3 years with average period of 9.3 months. The mode of injury was fall from height in 7 and motor vehicle accident in 2 patients. The average period of traction at hospital before the application of brace was 11 days (range 7 to 20 days). The time gap between admission of the patient to hospital and application of orthosis was taken for preparation of custom made orthosis. Usually orthosis was available by
Radiological union was seen to occur at 12 th week in 7 patients and 9th week in 2 patients and the brace was discarded at that time. Though the patients were taught to ambulate on crutches while on the orthosis, none did so. Pressure sores were seen around buttock and waist in two patients. These sores were superficial and healed with dressing. Complications like compartment syndrome, nerve palsies, breakage of orthosis, delayed union/nonunion, refracture did not occur. Only one patient had shortening 
of the limb by $1 \mathrm{~cm}$.( Figure 4) discarded at that time. Though the patients were taught to ambulate on crutches while on the orthosis, none did so. Pressure sores were seen around buttock and waist in two patients. These sores were superficial and healed with dressing. Complications like compartment syndrome, nerve palsies, breakage of orthosis, delayed union/nonunion, refracture did not occur. Only one patient had shortening of the limb by $1 \mathrm{~cm}$.( Figure 4)

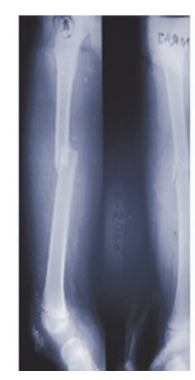

a.

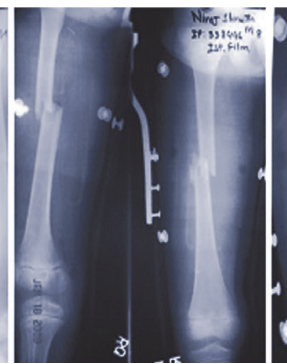

b.

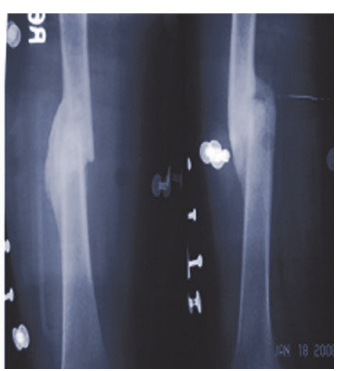

c.
Figure 4 Radiographs of a eight year old boy with transverse fracture shaft of femur

(a) on the day of injury, ( b) one week after application of brace (c) at 12 weeks follow up, showing union with overriding of fragments.

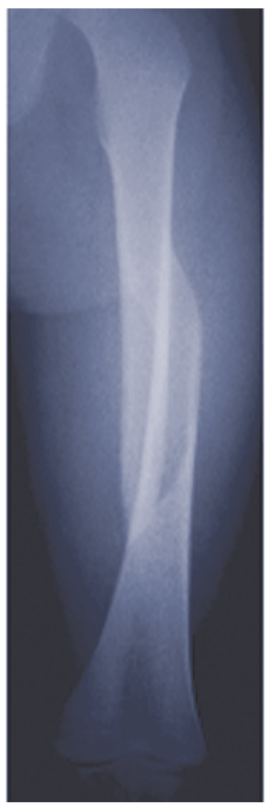

a

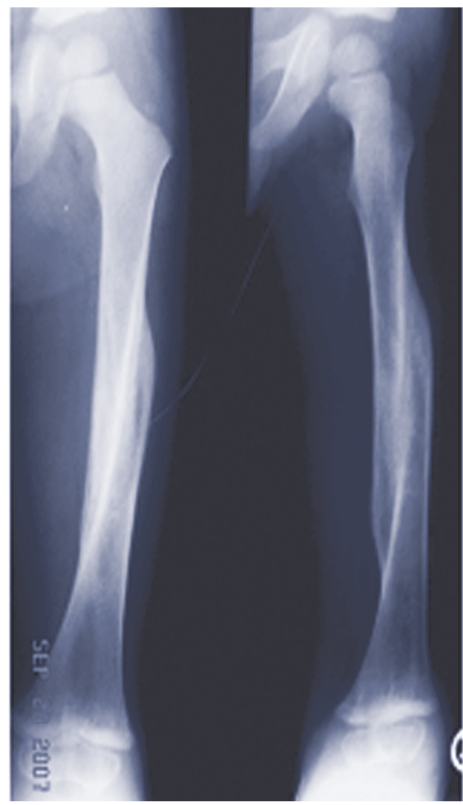

b.
Figure 5 Radiographs of a 4 year old boy (a) with fracture (b) at 3 months
There was no rotational malalignment of the limb in any of the patients. Figure 5 showing roentgenograms of our patients with complete union of fractured femur without any complications. Two patients with initial varus angulation of 10 degrees each did not get corrected after application of brace. Varus deformity persisted till last follow up (Figure 6).

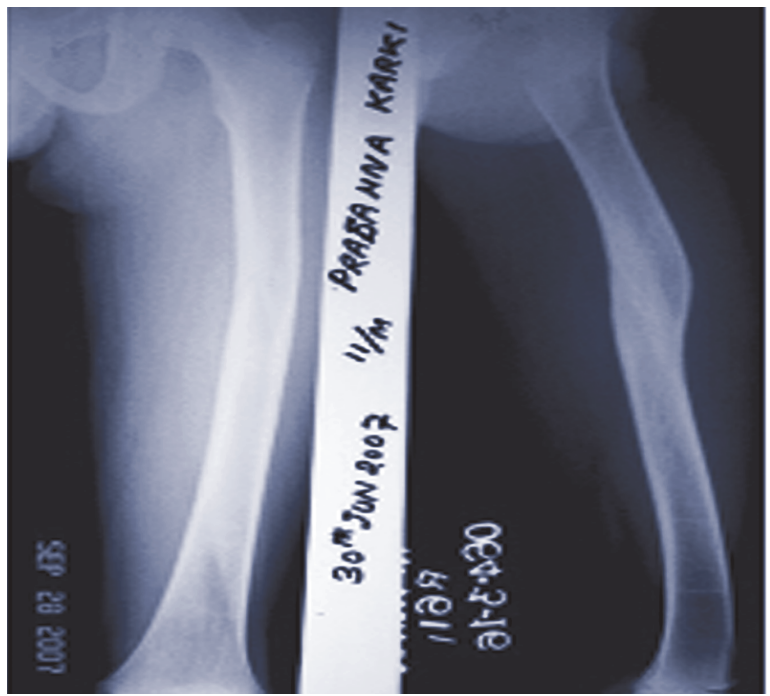

Figure 6 Radiograph at 2 years follow up of a patient showing 100 varus deformity following fracture shaft of femur at the age of 9 years

\section{Discussion}

The treatment of femoral fracture with cast bracing was more commonly used previously when the methods of internal fixation were not as advanced. Its advantages over other treatment methods in those days were early mobilization, prevention of knee stiffness and lessened hospital stay. Nowadays the trend is to treat older children's femur fractures operatively and younger children's nonoperatively ${ }^{3}$. But in a developing country like Nepal, where there are few centers equipped with facility and expertise for surgical treatment of femoral fracture, most of the children with femoral fracture undergo the traditional method of treatment (traction followed by spica cast), as it is already an established method of treatment. The hospital bed which is at a premium remains occupied for 3-6 weeks when a child undergoes treatment with this method. Cast bracing saves valuable hospital bed space and also avoids potential surgical complications in operative fixations like wound infection, osteonecrosis of femoral head, delayed union/nonunion, growth disturbance and a second operation for implant removal. Most of the reported use of braces in treatment of fracture femur dates back to seventies and eighties. 
It restricts mobilization and positioning in multiple trauma victims. Although rare, significant complications have been reported using this technique, including foot drop, skin loss, compartment syndrome, and malunion ${ }^{4}$. Cast pressure sores, especially in the lower back area, are possible. Inability to maintain proper alignment with spica casting is a concern, particularly with large or obese patients, adolescents, or very unstable fracture patterns. Excessive shortening is the most common early complication of spica casting ${ }^{5}$. Despite recent concerns of malunion, limb length inequality, and significant skin complications with the use of spica casts, this method is still considered by many to be the treatment of choice for younger age groups. ${ }^{5}$

Gross RH et $\mathrm{al}^{6}$ (1983) used cast bracing in seventy-two children and adolescents aged 5-19 years with femoral fractures. ${ }^{6}$ Results were generally comparable to those of more standard methods of treating a fractured femur in this age group. They noted that the varus and anterior angulation was not well controlled in proximal fractures and middle-third fractures showed more tendency toward shortening.

Hardy $\mathrm{AE}^{7}$ (1982) reported twenty-eight patients with femoral shaft fractures treated by early three piece cast brace application and ambulation. ${ }^{7}$ The common complications were femoral shortening and varus angulation but when bony union occurred, shortening did not exceed length lost from telescoping. In order to avoid shortening it was advised that if roentgenograms obtained in the week after cast brace application demonstrate unacceptable shortening, bed rest and skeletal traction should be instituted.

Mallo and colleagues concluded from a comparative study that there was no statistical difference between the malunion rate of children treated by traction-spica cast and traction-functional brace and the functional brace appears to be a good alternative for the treatment for femoral shaft fracture in children allowing an earlier ambulation than spica cast. ${ }^{8}$

Though internal fixation device has completely taken over other treatment modalities in adult femur fracture, the preliminary report of our study reflects that the cast bracing is still a better option for femur fracture in children. All the fractures healed completely without serious complications. The varus angulations in two patients were with in the acceptable range. Middle third transverse fractures of femur are known for shortening due to over-riding of fracture ends. We had shortening of $1 \mathrm{~cm}$ in an 8 year old boy with mid shaft transverse fracture at 6 months follow up. Fracture pattern in other 8 patients were either spiral or oblique. So the brace is still to be tested with more different patterns of femoral shaft fracture. Inability to walk on crutches by the patients was unexpected, and was probably due to difficulty in adapting to the brace in standing position. However we believe that a co-operative older child can be mobilized on crutches after clinical union of fracture. Inability of the brace to correct varus angulation seems to be its weakness. The rotational stability given by the brace was excellent. None of the cases had rotational deformity. The result so far is encouraging and seems that the orthotic treatment of fracture shaft femur is as good as the traditional method with distinct advantages of treating at home and gives opportunity to study school syllabus at home and may even allow co-operative older children to attend school. But it is still too early to draw any definite conclusion from this study as the number and variety of cases are limited.

\section{Conclusion}

The preliminary findings of the study with the use of this Orthosis in the treatment of fracture shaft of femur in children has proved to be as good as the traditional method with distinct advantages of treating at home and early mobilization with the brace on. Its success lies in the excellent application of basic orthotic principles.

Conflict of interest: The authors declare that they have no conflict of interests.

\section{References}

1. Kirby RM, Winquist RA, Hansen ST Jr.. Femoral shaft fractures in adolescents: A comparison between traction plus cast treatment and closed intramedullary nailing. J Pediatr Orthop. 1981; 1:193-197.

2. Rab GT, Grottkau BE..Operative treatment of children's fractures and injuries of the physis. In Szabo RM et al Chapman's Orthopedic Surgery 3rd ed. Philadelphia: Lippincott Williams \& Wilkins; 2001:41; 75-82.

3. Sanders JO, Browne RH, Mooney JF,et al Treatment of femoral fractures in children by pediatric orthopedists: results of a 1998 survey, J Pediatr Orthop. 2001; 21(4):436-41.

4. AAOS Bulletin. Femur fracture care frequent cause of lawsuit. February 2001 . http://www2.aaos.org/bulletin/ feb01/fline4.htm.

5. Anglen JO, Choi L,. Treatment Options in Pediatric Femoral Shaft Fractures. J Orthop Trauma. 2005;19:724-733

6. Gross RH, Davidson R, Sullivan JA, Peeples RE, Hufft R. Cast brace management of the femoral shaft fracture in children and young adults. J Pediatr Orthop. 1983; 3(5):572-82.

7. Hardy AE.Shortening and angulation of femoral shaft fractures treated by cast brace application and early ambulation Clin Orthop Relat Res. 1982; 168:139-43.

8. Malo M, Grimard G, Morin B.. Treatment of diaphyseal femoral fractures in children: a clinical study, Ann Chir. $1999 ; 53(8): 728-34$ 